\title{
Impacto ambiental en áreas marginales del río Colorado, provincia de Buenos Aires, Argentina ${ }^{1}$
}

\author{
Environmental impact in marginal areas of the Colorado River, \\ province of Buenos Aires, Argentina
}

\author{
Maira Scherger ${ }^{2}$ \\ María Patricia Rosel1 ${ }^{3}$ \\ Alicia María Campo ${ }^{4}$
}

\section{Resumen}

Las áreas marginales expuestas a la recurrencia de sequías -fenómenos climáticos extremos- actúan como catalizador en el proceso de desertificación. El desarrollo sostenible es definido como aquel que satisface las necesidades del presente sin comprometer las necesidades de las futuras generaciones, esto implica la relación inseparable entre la sostenibilidad de un recurso y su contexto económico y social. En el presente trabajo se reconocen las transformaciones espaciales en el Valle Bonaerense del río Colorado a partir de la puesta en valor del río Colorado como recurso y se identifican y seleccionan los componentes ambientales impactados por las actividades agropecuarias. El agua, recurso natural que ofrece el río, ha sido el elemento organizador de este espacio que se caracteriza por la producción agrícola. En las últimas décadas las oportunidades económicas internacionales del mercado, han favorecido el incremento de superficies para tal fin. En

Proyecto de investigación estrategias de gestión y formación para el desarrollo local en espacios urbanos, periurbanos y rurales del suroeste bonaerense. (24/G056) (2009-2012). Proyecto subsidiado por la Secretaría de Ciencia y Tecnología de la Universidad Nacional del Sur.

2 Licenciada en Geografía. Filiación Institucional: Universidad Nacional del Sur. País: Argentina. Correo electrónico: mascherger@ gmail.com.

3 Magíster en Geografía. Universidad Nacional del Sur. Filiación Institucional: Profesora adjunta exclusiva. Departamento de Geografía y Turismo, Universidad Nacional del Sur. País: Argentina. Correo electrónico: prosell@uns.edu.ar

4 Doctora en Geografía. Universidad Nacional del Sur, Argentina. Profesora asociada, Departamento de Geografía y Turismo, Universidad Nacional del Sur. Filiación Institucional: Profesora titular, Facultad de Ciencias Humanas, Universidad Nacional del Centro de la Provincia de Buenos Aires. Investigadora adjunta. Consejo Nacional de Investigaciones Científicas y Técnicas. País: Argentina. Correo electrónico: amcampo@uns.edu.ar 
consecuencia, el uso intensivo del suelo y la falta de adecuadas políticas de planificación impactan negativamente generando procesos de degradación de suelos que atentan el desarrollo sostenible de la región.

Palabras clave: Degradación de suelos, desarrollo sostenible, impacto ambiental.

\section{Abstract}

Marginal areas exposed to the recurrence of drought, extreme weather events, act as a catalyst in the process of desertification. This paper recognizes transformations in the Buenos Aires Valley of the Colorado River from the valorization of the Colorado River as a resource and are identified and selected environmental components impacted by agricultural activities. Concludes that natural water supply, coupled with economic opportunities in the market have led to the agricultural intensification in the Bonaerense Valley of the River. As a result of this process, the overuse of soil is linked to the increase in salinity, increased erosion and the progressive decline in the quality of agricultural land.

Keywords: Degradation of soils, sustainable development, environmental impact. 


\section{Introducción}

Los problemas que afectan la conservación del ambiente, en especial si devienen de la explotación de recursos naturales como el suelo, son múltiples y complejos. La falta de una adecuada planificación regional de estos recursos involucra, en gran parte, el riesgo de pérdida de los servicios ecológicos esenciales, que impacta negativamente en la producción agropecuaria y afecta directa o indirectamente a la sociedad vinculada con la actividad.

Con este estudio se pretende reconocer la relación que existe entre el uso intenso del suelo agrícola y el impacto de las condiciones climáticas extremas, como el caso de la sequía. Se trata de un aporte a la generación de los conocimientos necesarios que permitan diagnosticar los problemas naturales y sociales que condicionan el modo de aprovechamiento de este recurso.

En el Informe Brundtland (Soler, 1997) se utilizó por primera vez el término desarrollo sostenible (o desarrollo sustentable), definido como aquel que satisface las necesidades del presente sin comprometer las necesidades de las futuras generaciones. Esto implica la relación entre sostenibilidad de un recurso con el contexto económico y social de desarrollo:

Para conseguir un desarrollo sostenible y equilibrado, compatible con la conservación de nuestro medio natural, es preciso aplicar un conjunto de principios básicos que informan la gestión en materia de protección del medio ambiente. De ellos destacan el principio de protección y el de la corrección de la contaminación o deterioro causados, internalizando los costes de la producción. (Conesa, 1997, p. 8).

El avance tecnológico agropecuario ocurrido en los últimos diez años en la región pampeana, ha facilitado incorporar nuevas tierras marginales, entre ellas las tierras del sudoeste bonaerense. La intensificación agrícola llevada a cabo por los productores causó mayor presión sobre el suelo y la vegetación nativa, ocasionando un proceso de degradación y disminución paulatina de la productividad.

El tipo de investigación está orientado por un marco conceptual y metodológico, en el que se concede particular importancia al impacto entre las interacciones de los fenómenos pertenecientes al medio físico, a la agroproducción y la estructura socioeconómica. La implementación de técnicas de evaluación de impacto ambiental orienta las medidas preventivas o correctivas, a fin de posibilitar estrategias de desarrollo basadas en un manejo integrado. Finalmente, las discusiones sobre los resultados nos permiten plantear las conclusiones y sugerir propuestas.

\section{Marco conceptual}

Una visión general de la situación de la agricultura en el mundo, demuestra el interés creciente por parte de la sociedad 
de vivir en espacios ambientalmente saludables, lo que conlleva, por supuesto, la voluntad de reconocer al recurso suelo como un elemento estratégico de desarrollo, que desempeña un papel básico para la supervivencia de ecosistemas y también para el desarrollo de las actividades humanas. Por su función ambiental, el suelo sostiene la actividad y la producción biológica, regula el flujo de agua y solutos, filtra y amortigua los materiales orgánicos e inorgánicos.

La visión actual de la marginalidad está vinculada con la acción no controlada del hombre, hecho que genera complejos conflictos en la productividad local. En consecuencia, surge la necesidad de conocer los impactos ambientales negativos, que se manifiestan como alteraciones desfavorables en el medio o en alguno de sus componentes (Estevan, 1989).

(...) hay impacto ambiental cuando una acción o actividad produce una alteración, favorable o desfavorable, en el medio o en alguno de los componentes del medio. Esta acción puede ser un proyecto de ingeniería, un programa, un plan, una ley o una disposición administrativa con implicaciones ambientales. (Conesa, 1997, p. 25).

Un instrumento decisivo para evitar el deterioro ambiental y dar solución adecuada a los distintos problemas que plantean los proyectos o acciones sobre el entorno, consiste en la aplicación de la Evaluación del Impacto Ambiental (EIA).

\subsection{Localización}

El Valle Bonaerense del río Colorado (VBRC) se encuentra ubicado en el sudoeste de la provincia de Buenos Aires. El área de estudio se extiende entre los paralelos de $39^{\circ} 10^{\prime}$ y $39^{\circ} 55^{\prime}$ de latitud Sur y los meridianos de $62^{\circ} 05^{\prime}$ y $63^{\circ}$ $55^{\prime}$ de longitud Oeste (Figura 1). Comprende los partidos de Villarino (donde se hallan emplazadas las localidades de Mayor Buratovich, Hilario Ascasubi y Pedro Luro), y de Patagones (en el que están situadas las localidades de Juan A. Pradere, Igarzábal y Villalonga), los cuales están limitados hidrográficamente por el río Colorado.

Se accede por la Ruta Nacional $\mathrm{N}^{\circ} 3$ que los vincula con la Patagonia y con el Ferrocarril Sur, además conecta la región con el resto del país. La Corporación de Fomento del Río Colorado - CORFO Río Colorado- posee jurisdicción sobre 530.419 has de los partidos mencionados anteriormente. De esta manera al partido de Patagones le corresponden 211.031 has y al partido de Villarino 319.388 has. De este total, 137.565 has se hallan empadronadas bajo concesión de riego.

\subsection{Características ambientales del área}

Topográficamente, la región se ubica en la porción meridional de la llanura pampeana, de condiciones naturales homogéneas caracterizadas por ausencia de fuertes contrastes en su relieve, con marcadas diferencias climáticas entre el norte del partido de Villarino hasta el 
Figura 1. Ubicación del área de estudio.

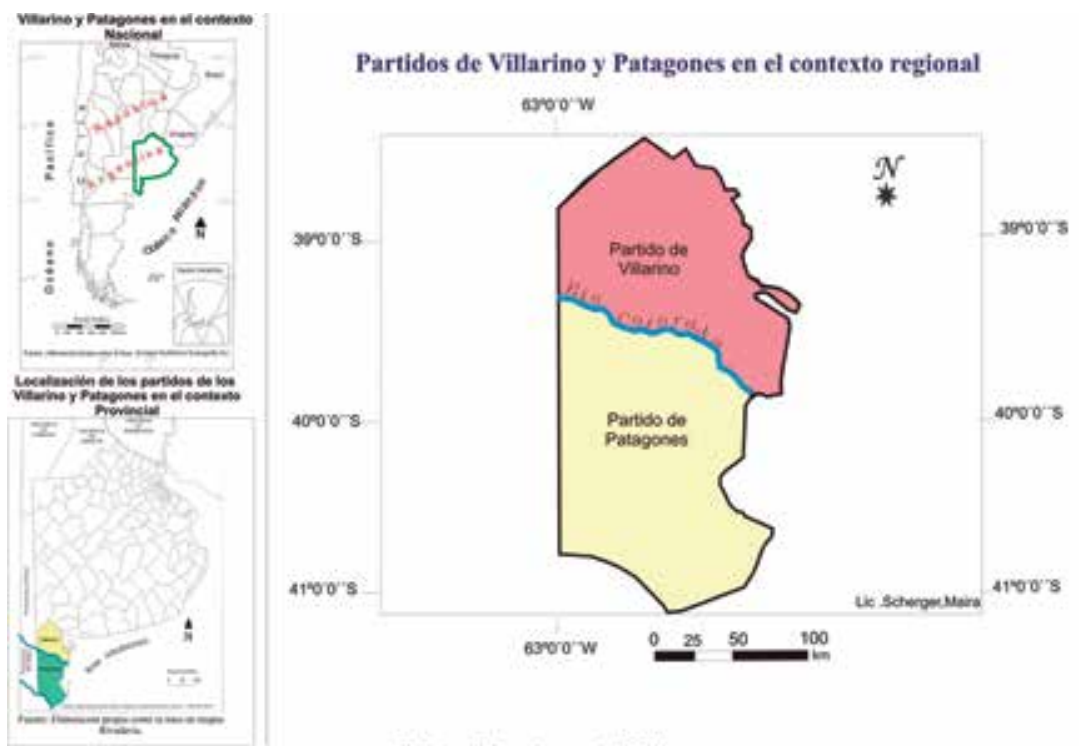

Fuente: Elaboración propia (2012)

Figura 2. Los suelos y vegetación. Partidos de Villarino y Patagones.

\section{Los suelos y vegetación en el Valle Bonaerense del Rio Colorado, provincia de Buenos Aires, Argentina}
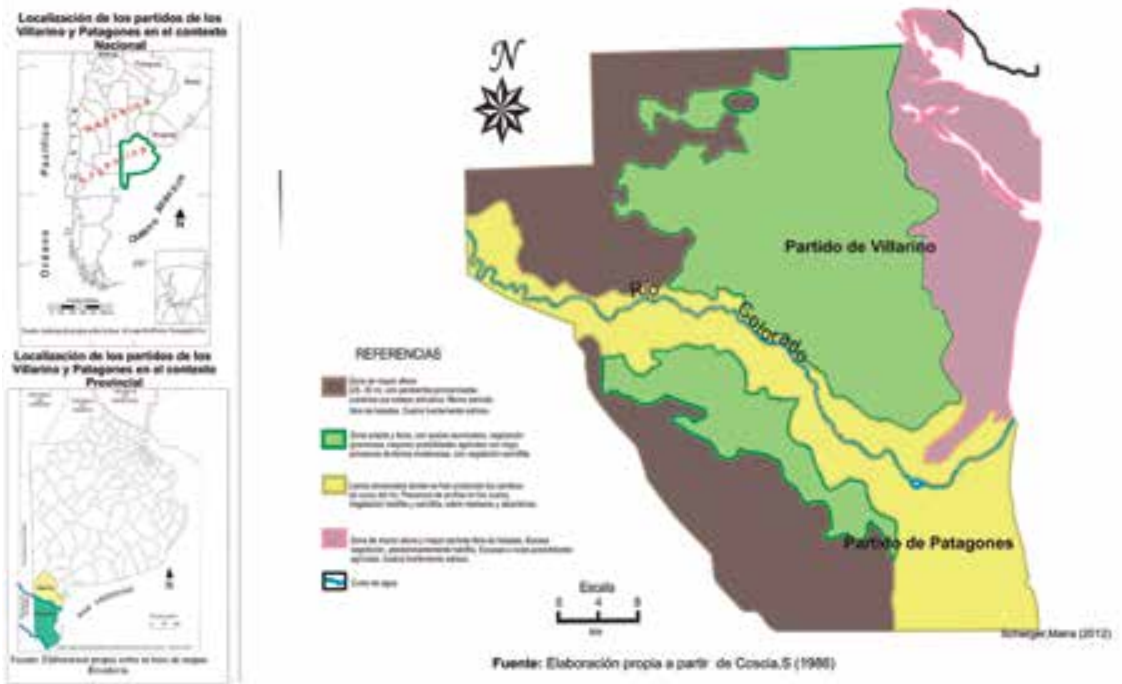

Fuente: Elaboración propia a partir de Coscias, S. (2012) 
límite sur del partido de Patagones. En esta zona se inicia la meseta patagónica cortada por los ríos Colorado y Negro (Capelli, 1994).

Los suelos de la región son relativamente profundos y con una débil diferenciación de horizontes en donde abunda la fase arenosa.

Los rasgos de continentalidad, exceptuando sectores costeros, evidencian amplias fluctuaciones en las temperaturas y precipitaciones durante el transcurso del año. Presenta como característica esencial una gran variedad en la condiciones del tiempo, hecho que se manifiesta en todas las estaciones del año. Las lluvias insuficientes hacen que se incluya dentro de los climas subhúmedos, variedad de clima templado, llamado de transición (Capelli, 1994).

El análisis del balance hídrico anual para el período 2000-2009 (Figura 3), corrobora el carácter seco de la región, ya que la evapotranspiración potencial refleja un promedio para el período de 776,90 $\mathrm{mm}$ anuales, ante un registro de precipitaciones de 436,90 mm. La deficiencia, por lo tanto, es de $288,05 \mathrm{~mm}$ y se extiende desde octubre hasta junio. Las altas temperaturas estivales se combinan con la acción de los vientos dominantes del Norte, Noreste y Oeste, cuya mayor frecuencia y velocidad provocan un au-

Figura 3. Balance hídrico período 2000-2009.

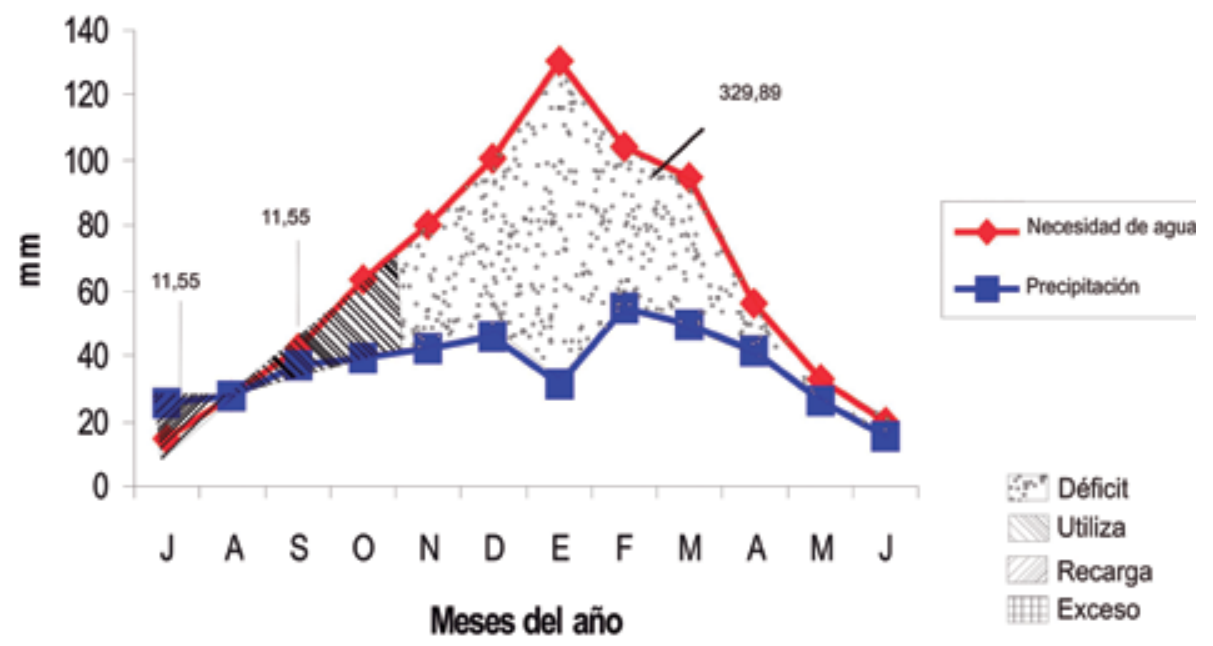

Fuente: Elaboración a partir de datos brinmdados por el INTA EEA Hilario Ascasubi (2010) 
mento en la evapotranspiración. Durante el mes de julio se repone la humedad del suelo $(11,55 \mathrm{~mm})$, la que se utiliza en el mes de agosto y octubre (11,55 mm). En octubre se inicia el déficit.

\subsection{Proceso de transformaciones espaciales en el área de estudio}

Las manifestaciones de intervención en el Valle Bonaerense del río Colorado se reconocen a partir de la llegada de los primeros colonos quienes valoraron las tierras con fines agrícolas. Uno de los pioneros, Pedro Luro, se estableció en 1882 en cercanías de la bifurcación del río entre los brazos "Grande y Chico", introdujo el ganado bovino y ovino y las primeras herramientas de labranza. Si bien constituye una primera intervención del medio, este período no implicó modificaciones sustanciales en el paisaje (Coscia, 1988).

En 1884, con la finalidad de incorporar el regadío al establecimiento, se abrió el canal San Adolfo. En pocos años se convirtió en uno de los modelos de explotación de la zona, a partir de los cultivos de alfalfa, con excelente calidad de semillas, de frutales, vid y ganado.

Con la llegada del ferrocarril a Carmen de Patagones, en 1921, se afincaron los primeros inmigrantes provenientes de Europa. Estos se instalaron como mano de obra en los establecimientos existentes y otros se radicaron en las cercanías de las estaciones ferroviarias, dando origen a las localidades de Mayor Buratovich y Villalonga.
Todas estas intervenciones se desarrollan a partir de condiciones económicas favorables, y, alrededor de 1940, se manifiestan en un crecimiento sostenido de la actividad frutihortícola.

A la expansión agrícola se suma la ampliación de la red de riego, por lo tanto en este período se manifiestan los primeros rasgos de salinización de los suelos. Desde 1960 hasta el presente, CORFO Río Colorado cumple la función de gestionar y supervisar los problemas relacionados con el riego y el desarrollo integral y regional del área.

\subsection{Selección y valoración de los componentes ambientales}

Se identifican y seleccionan los componentes ambientales pertenecientes al medio físico, susceptibles de recibir el impacto y las consecuencias de las acciones productivas. La selección de los mismos se obtiene de la bibliografía consultada, entrevistas, análisis de mapas satelitales multitemporales y balances hídricos. De este modo se pudo simplificar la información para ser aplicada en la matriz de impacto.

Los indicadores seleccionados son suelo, agua, condiciones climáticas y paisaje. Los componentes ambientales por considerar son aire, suelo, agua, clima, biodiversidad y paisaje. El elemento atmósfera resulta ser un indicador ambiental relevante, pero, para este caso, no fue posible su aplicación dada la dificultad de obtención de datos confiables. 
En cuanto a la distribución de la diversidad biológica actual, se pone énfasis en que la misma es el resultado de los procesos evolutivos, biogeográficos y ecológicos a lo largo del tiempo. Su existencia, conservación y evolución dependen de los factores ambientales que la hacen posible aportando en gran medida los servicios ecológicos básicos para garantizar el bienestar y equilibrio de la vida.

Durante los últimos años se observa la pérdida cada vez más acelerada de la biodiversidad. Fundamentalmente los proyectos agrícolas y ganaderos que implican el desmonte de tierras, con el consecuente desplazamiento de la vida silvestre a favor de la introducción de la ganadería, el uso de fertilizantes, y pesticidas, y la eliminación de tierras naturales a favor de la inundación para riego, son las prácticas más amenazantes.

También se evalúa el carácter del paisaje, a partir de las cualidades intrínsecas que posee el área de estudio. Por lo tanto, se consideran los componentes escénicos visibles que se han modificado a lo largo del transcurso de la ocupación del territorio y también los procesos ecológicos que responden a interrelaciones no directamente perceptibles, y que encierran la clave de la estructura y apariencia del mismo. De manera que, en el área de estudio, los componentes ambientales (biodiversidad y paisaje) se analizan teniendo en cuenta todas las acciones impactantes mencionadas.

\subsection{Identificación de acciones que puedan causar impactos}

Se resumen las acciones más relevantes que manifiestan una posible alteración.

\section{Usos y cobertura de suelos}

En los partidos de Villarino y Patagones se distinguen dos áreas mixtas importantes, a saber: un área de riego incluida en el Valle Bonaerense del Río Colorado y otra de secano, en Patagones. Dumrauf (2008, p. 46) anota:

La expansión de la frontera agrícola se produjo por diversas razones. Las áreas de secano tanto de Villarino como de Patagones fueron pobladas por productores que vinieron desde otras zonas del país, que debieron desplazarse debido a procesos de erosión eólica, lo que se llamó periodo de éxodo pampeano. La provincia de Buenos Aires tenía mucha tierra fiscal en estos partidos que fue otorgada a estos productores. La mayoría de ellos eran descendientes de alemanes del Volga, con una cultura triguera muy arraigada, la cual hizo que se empezara a desmontar y a sembrar trigo.

Esta evolución se analizó sobre la base de los usos y cobertura de suelos (Tabla 1).

Durante el año 1975 la superficie del partido de Villarino contaba con 406.176 has cultivadas representando el $40 \%$ de la superficie. Del mismo, el $12 \%$ correspondía al área de riego y el $28 \%$ restante, al área de secano. Para 1987, 
Tabla 1. Uso y cobertura de suelos (en hectáreas) en el partido de Villarino

\begin{tabular}{|c|c|c|c|c|c|c|}
\hline & 1975 & $\%$ & 1987 & $\%$ & 2005 & $\%$ \\
\hline Cadena de médanos & 136.416 & 13 & 130.958 & 13 & 127.064 & 13 \\
\hline Cuerpos de agua & 3.469 & 0,4 & 6.179 & 0.5 & 7.133 & 0.7 \\
\hline Tierras agrícolas de riego & 122.644 & 12 & 113.871 & 11 & 151.279 & 15 \\
\hline Tierras agrícolas de secano & 283.532 & 28 & 303.742 & 30 & 344.247 & 34 \\
\hline Monte & 295.566 & 29 & 287.757 & 28.5 & 249.902 & 24.8 \\
\hline Pastizales halófilos & 148 & 14,6 & 128.819 & 13 & 95.538 & 9.5 \\
\hline Salina & 12.89 & 1 & 6.729 & 1 & 9.267 & 1 \\
\hline Slitral & 17.780 & 2 & 20.118 & 2 & 20.570 & 2 \\
\hline
\end{tabular}

Fuente: Dumrauf (2008)

el área agrícola de secano se expande un $1 \%$, y el área de riego disminuye con respecto al periodo anterior en un $1 \%$. Las tierras bajo riego al noroeste del partido, conformaban un cinturón hortícola que fue absorbido por la zona de secano. En cuanto al año 2005, la zona agrícola creció un $49 \%$, extendiéndose tanto el área de riego y de secano un $4 \%$ cada una con respecto al año 1987.

Se puede observar en la tabla 2 que el área agrícola del partido de Patagones, se ha extendido sustancialmente.

\section{Agricultura}

La economía del VBRC se basa en la producción agropecuaria (cereales, oleaginosos y ganadería vacuna), con una fuerte especialización en el subsector hortícola (cultivo de cebolla). Se explotan, a su vez, la apicultura y, en menor medida, frutas secas y fina. Durante la década de 1990, una fuerte expansión sustentada en las oportunidades comerciales asociada a la conformación del Mercado Común del Sur MERCOSUR, trajo consigo un incremento en la superficie, producción y

Tabla 2. Uso y cobertura de suelos en hectáreas. Partido de Patagones

\begin{tabular}{|c|c|c|c|c|c|c|}
\hline & 1975 & $\%$ & 1987 & $\%$ & 2005 & $\%$ \\
\hline Tierras agrícolas de riego & 3.196 & 0.2 & 2.196 & 0.2 & 1.374 & 0.1 \\
\hline Tierras agrícolas de Secano & 78.726 & 5.4 & 182.676 & 13.5 & 190.255 & 14 \\
\hline Monte & 371.1 & 25.7 & 471.864 & 34.8 & 636.101 & 46.7 \\
\hline Pastizales halófilos & 944.59 & 65.4 & 645.979 & 47.6 & 479.950 & 35.3 \\
\hline Salina & 3.104 & 0.2 & 3.139 & 0.2 & 3.133 & 0.2 \\
\hline Salitral & 44.156 & 3.1 & 51.597 & 3.8 & 49.904 & 3.7 \\
\hline SUPERFICIE DEL PARTIDO & \multicolumn{7}{|c|}{1.402 .639} \\
\hline
\end{tabular}

Fuente: Dumrauf (2008) 
productividad del cultivo de cebolla que se elevan significativamente durante el periodo 1990-1991.

Durante el período 2009/2010 se ha sembrado una superficie de 13.457 has; la fuerte especialización en el subsector hortícola por el cultivo de cebolla evidencia esta situación.

\section{Ganadería}

La ganadería es la actividad que mejor se adapta ecológicamente en el partido de Villarino. Sin embargo, de acuerdo con los datos proporcionados por el Instituto Nacional de Tecnología Agropecuaria INTA, los rodeos de cría han sufrido una importante reducción asociada a la sequía, ya sea por éxodo o mortandad. El stock ganadero más alto registrado fue en 2005 con 381.000 cabezas, mientras que para 2009 se contabilizaron 148.491 cabezas, lo que equivale a un $40 \%$ menos que en el año 2005 (INTA, 2009).

\section{Desmonte}

El monte xerófilo ha sido un recurso muy valioso desde el punto de vista histórico y económico como fuente de combustible y aprovechamiento de maderas duras. El avance de la frontera agrícola sobre el monte nativo en el partido de Patagones, determinó que entre 1975 y el 2009 un total de 432.280 has fueran taladas a favor del recurso suelo (Tabla 3 ).

Durante este período, la tasa media anual de pérdida de biomasa fue del $3,7 \%$, lo que causó un deterioro del suelo produ- ciendo cambios edáficos, por la desaparición del horizonte fértil expuesto a la erosión eólica. Se produjo de esta manera

Tabla 3. Tasa de reducción anual de monte nativo

\begin{tabular}{|c|c|c|}
\hline Año & $\begin{array}{c}\text { Superficie } \\
\text { de monte en } \\
\text { hectáreas }\end{array}$ & $\begin{array}{c}\% \\
\text { Monte }\end{array}$ \\
\hline 1975 & 911.171 & 65 \\
\hline 1987 & 682.367 & 49 \\
\hline 1999 & 554.138 & 40 \\
\hline 2002 & 524.629 & 37 \\
\hline
\end{tabular}

Fuente: Pezzola (2004)

la aridización de los mismos, siendo muy difícil la recuperación de la comunidad original (Pezzola, 2004).

En sus comienzos, los desmontes fueron exclusivamente manuales; actualmente el empleo de maquinarias incrementa rápidamente la incorporación de la superficie disponible, así son cada vez menores las superficies cubiertas por el monte autóctono que son empleadas como tierras de pastoreo.

\section{Red de riego y drenaje}

El riego en los partidos de Villarino y Patagones, comprende las márgenes oeste y este, respectivamente, del Río Colorado. Sobre un total de 535.000 has que integran ambos partidos, se incluyen bajo riego 135.000 has, lo cual manifiesta un ritmo importante de crecimiento en relación con el año 2001, cuando se destinaban al riego tan solo 80.000 has. Desertificación 
Tanto el avance sobre el ecosistema nativo, como el posterior avance inadecuado de la producción agrícola-ganadera, han favorecido la pérdida de estructura y fertilidad de los suelos jóvenes de esta región.

Entre 1980-1990, el aumento de las precipitaciones sobre la media histórica generó un corrimiento de las isohietas hacia el suroeste. Este pulso húmedo generó gran expectativa propiciando la intensificación agrícola en los productores y causó presión sobre el suelo y la vegetación nativa (Figuras 4 y 5).

Estudios efectuados por INTA (2010) de la localidad Hilario Ascasubi, durante el período comprendido entre julio de 2007 a julio de 2009 , determinaron que los suelos del partido de Patagones carecían de la cobertura vegetal y estaban altamente expuestos al efecto erosivo del viento.

Figura 4. Distribución de isoyetas. Partidos de Villarino y Patagones.

Período 1971-2000.

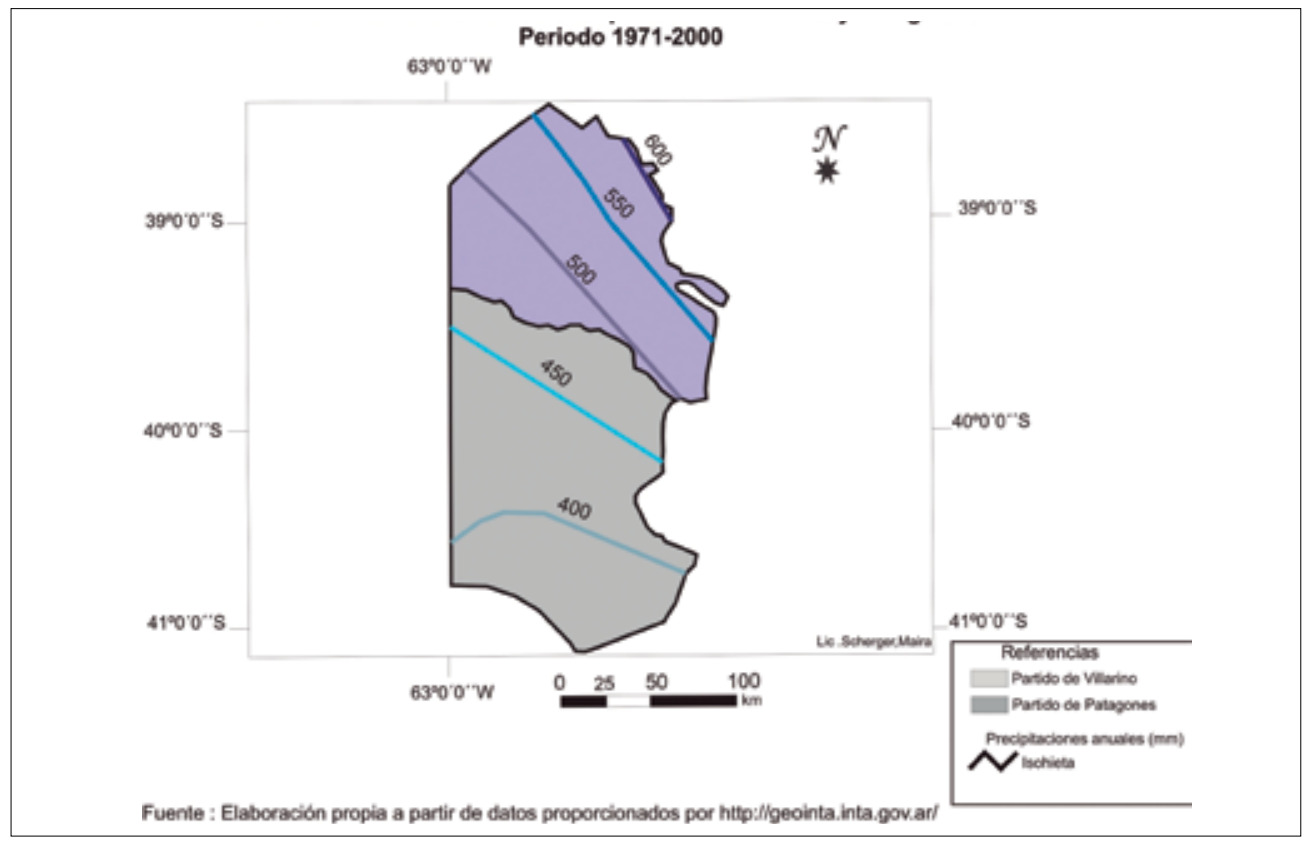

Fuente: Elaboración propia a partir de datos proporcionados por http://geointa.inta.gov.ar/Período 1971-2000. 
Figura 5. Distribución de isoyetas. Partidos de Villarino y Patagones. Año 2009.

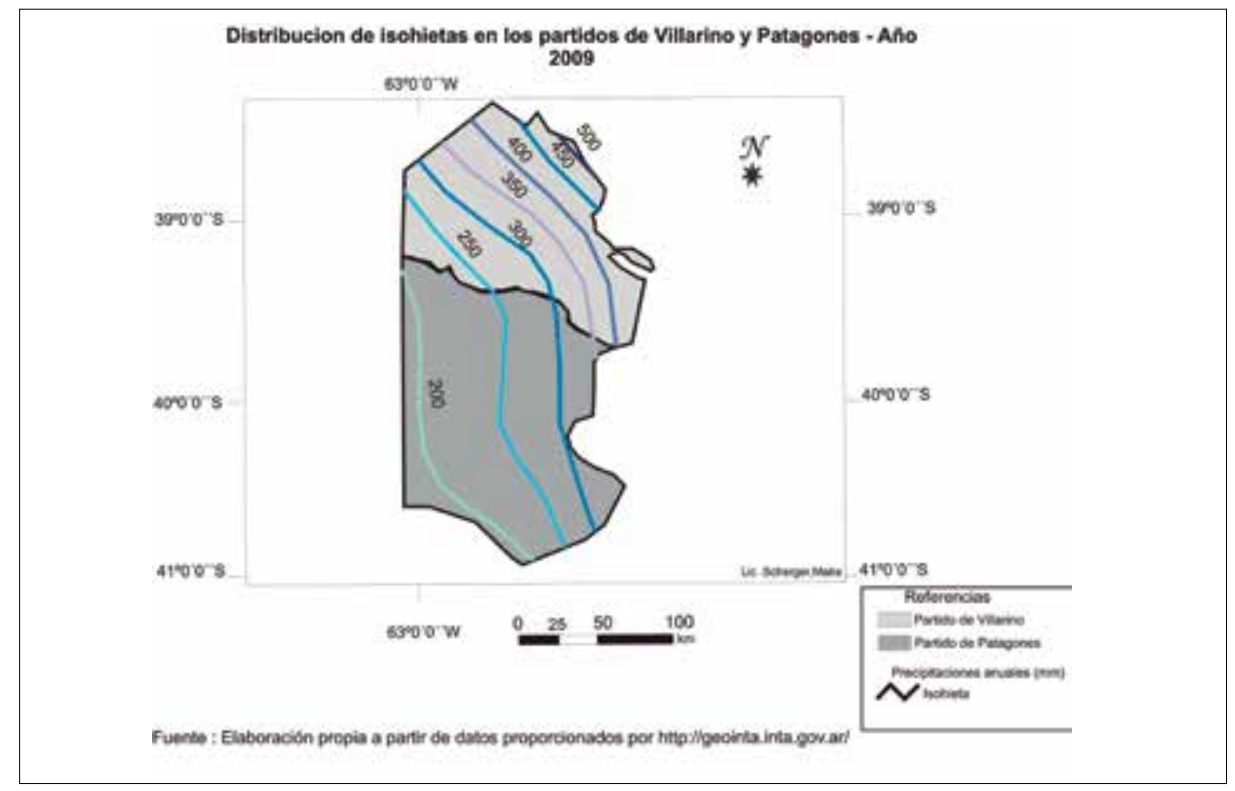

Fuente: Elaboración propia a partir de datos proporcionados por http://geointa.inta.gov.ar7

Tabla 4. Cantidad de hectáreas erosionadas en el partido de Patagones

\begin{tabular}{|c|c|c|}
\hline \multicolumn{2}{|c|}{ Superficie en hectáreas } \\
\hline Partido & Área Mixta & Suelos erosionados \\
\hline 1.360 .717 & 683.463 & \\
& & \\
\hline G1 Leve a moderado & 47.337 hectáreas & \\
\hline G2 Moderado a grave & 219.204 hectáreas & \\
\hline G3 Grave a severo & 126.970 hectáreas & \\
\hline TOTAL & 393.511 hectáreas & \\
\hline
\end{tabular}

Fuente: INTA, localidad de Hilario Ascasubi (2009) 


\section{Incendios}

Teniendo en cuenta la variación anual de las temperaturas, se observan en ambos partidos características de ambientes continentales. Los vientos secos durante la estación estival determinan una evapotranspiración muy elevada, lo que ocasiona situaciones de estrés hídrico. Es así que estas condiciones climáticas provocan un efecto muy elevado en las formaciones vegetales xerófilas, facilitando de esta manera procesos combustivos.

Es importante tener en cuenta que el fuego, debido a su magnitud, no solamente afecta alambrados sino también viviendas, herramientas agrícolas, ganado, entre otros, lo que genera un alto impacto negativo para la economía. No obstante, se reconoce que el origen del incendio en varias oportunidades ha sido intencional o en su defecto por prácticas inadecuadas de la población.

El menor riesgo de incendios se presenta en las áreas de cultivos intensivos localizadas en el Valle Bonaerense del Río Colorado, en el Noreste y en la franja central de dicho distrito. Estos son sectores menos propensos a sufrir incendios, situación que puede asociarse con la mayor humedad ambiental propia de zonas con riego artificial (Pezzola, 2002).

\section{Actividad industrial y comercial}

Conforme el incremento de hectáreas para la actividad agropecuaria, en es- pecial relacionado con el cultivo de cebolla, surgen los galpones de empaque. En la etapa de acondicionamiento de la cebolla se producen grandes volúmenes de desecho compuesto por restos de hojas, tallos, raíces, catáfilas y bulbos de descarte, arrojados alrededor de los galpones.

A su vez, comercios relacionados con esa actividad y otras afines, generan un significativo crecimiento económico y por lo tanto radicación de nuevas familias en las localidades mencionadas.

De acuerdo con datos publicados por la Fundación Barrera Patagónica, en el año 2004 se habilitan 76 galpones de empaque, un número menor que en 1999.

Según informes proporcionados por el INTA, en el año 2009 se desechó entre el 3\% a 5\% del total de la cosecha de cebolla, por problemas de calidad y sanidad. La eliminación de los residuos se realiza sin un tratamiento previo, generalmente bajo la práctica de la quema en el campo, o arrojados en terrenos baldíos generando montículos. Surgen entonces neoecosistemas y microrrelieves que cambian los patrones de escurrimiento de los excedentes hídricos.

En todo sistema se produce un rediseño ecogeográfico del paisaje, es decir que aparecen nuevas estructuras biofísicas, denominadas microrrelieves y neoecosistemas. Estas se generan por excavaciones, rellenos y disposición de residuos y por el reemplazo de la vegetación autóctona degradada e invadida por especies 
oportunistas de gran competitividad. Presentan degradación ambiental y son vulnerables con relación a la incidencia de acciones humanas, constituyendo así zonas de riesgo

\section{Paisaje}

Desde las primeras transformaciones espaciales hasta el presente, el área ha sido afectada por impactos ambientales que han fragmentado el paisaje del valle con la consecuente pérdida de las características propias originales. Esto pone de manifiesto impactos acumulados a lo largo de su historia

\section{Aplicación de la matriz de importancia del impacto}

Con los indicadores seleccionados y los factores más relevantes se pudo simplificar la información por aplicar en la matriz de impacto. Para esto se recurrió al método de Conesa (1997), una matriz de relaciones en cuyas columnas se indican las actividades o acciones y en las filas, los factores ambientales discriminados según el medio biofísico y social. Con ella, se intenta hacer una síntesis sobre los efectos medioambientales de las acciones, así también como conocer la intensidad y magnitud de los impactos. Se establece un índice ponderal para cada factor, expresado en unidades de importancia (UIP). La cantidad de unidades asignada al total de los factores ambientales, presenta un valor de 100 , distribuidos en los 12 factores considerados.

La importancia del impacto para cada factor queda representada por un número que se deduce en función del valor asignado a los símbolos considerados en la siguiente fórmula:

La importancia del impacto varía entre 13 y 100 . Estos valores pueden ser positivos o negativos. En la presente investigación dicha valoración fue negativa, pues solo fueron considerados impactos de este tipo (Tabla 5).

$$
I=+l-\left[\left(3^{*} 1\right)+\left(2^{*} E X\right)+M O+P E+R V+S I+A C+E F+P R+M C\right] * U I P
$$

Donde: I: intensidad; EX: extensión; MO: momento; PE: persistencia; RV: reversibilidad; SI: sinergia; AC: acumulación; EF: efecto; PR: periodicidad; MC: recuperabilidad y finalmente UIP que equivale a las unidades de importancia asignadas a cada factor impactado.

Fuente: Conesa (1997) 
Tabla 5. Importancia del impacto

\begin{tabular}{|c|c|c|c|}
\hline \multicolumn{2}{|l|}{$\begin{array}{c}\text { INTENSIDAD (I) } \\
\text { (Grado de destrucción) }\end{array}$} & \multicolumn{2}{|l|}{$\begin{array}{l}\text { EXTENSIÓN (EX) } \\
\text { (Área de influencia) }\end{array}$} \\
\hline Baja ......... & 1 & Puntual .... & 1 \\
\hline Media .... & 2 & Parcial . & 2 \\
\hline 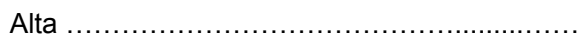 & 4 & Extenso ............... & 4 \\
\hline 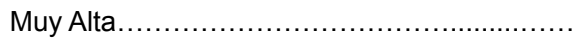 & 8 & & \\
\hline \multicolumn{2}{|l|}{$\begin{array}{c}\text { MOMENTO (MO) } \\
\text { (Plazo de manifestación) }\end{array}$} & \multicolumn{2}{|l|}{$\begin{array}{c}\text { PERSISTENCIA (PE) } \\
\text { (Permanencia del efecto) }\end{array}$} \\
\hline Largo plazo & 1 & Fugaz $\ldots \ldots \ldots \ldots \ldots \ldots \ldots \ldots \ldots$ & 1 \\
\hline Medio plazo & 2 & Temporal ...... & 2 \\
\hline Inmediato......$\ldots \ldots$ & 4 & Permanente .................... & 4 \\
\hline \multicolumn{2}{|l|}{ REVERSIBILIDAD (RV) } & \multicolumn{2}{|l|}{$\begin{array}{c}\text { SINERGIA (SI) } \\
\text { (Regularidad de la manifestación) }\end{array}$} \\
\hline Corto plazo & 1 & Sin sinergismo $\ldots \ldots \ldots \ldots \ldots \ldots \ldots \ldots$ & 1 \\
\hline Medio plazo & 2 & Sinérgico ............ & 2 \\
\hline Irreversible $\ldots \ldots \ldots \ldots \ldots \ldots \ldots$ & 4 & Muy sinérgico $\ldots \ldots \ldots \ldots \ldots \ldots \ldots$ & 4 \\
\hline \multicolumn{2}{|l|}{$\begin{array}{c}\text { ACUMULACIÓN (AC) } \\
\text { (Incremento progresivo) }\end{array}$} & \multicolumn{2}{|l|}{$\begin{array}{c}\text { EFECTO (EF) } \\
\text { (Relación causa-efecto) }\end{array}$} \\
\hline 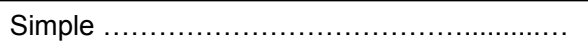 & 1 & Indirecto (secundario) . .................... & 1 \\
\hline Acumulativo ..................... & 4 & Directo ......... & 4 \\
\hline \multicolumn{2}{|l|}{$\begin{array}{c}\text { PERIODICIDAD (PR) } \\
\text { (Regularidad de la manifestación) }\end{array}$} & \multicolumn{2}{|l|}{$\begin{array}{c}\text { RECUPERABILIDAD (MC) } \\
\text { (Reconstrucción por medios humanos) }\end{array}$} \\
\hline Irregular o aperiódico y discontinuo................. & 1 & Recuperable de manera inmediata .......... & 1 \\
\hline Periódico .. & 2 & Recuperable a medio plazo . & 2 \\
\hline Continuo $\ldots . .$. & 4 & $\begin{array}{l}\text { Mitigable ............ } \\
\text { Irrecuperable ...... }\end{array}$ & $\begin{array}{l}4 \\
8\end{array}$ \\
\hline
\end{tabular}

Fuente: Conesa (1997)

Naturaleza: Carácter perjudicial (-) o beneficioso $(+)$ del impacto.

Intensidad (I): expresa el grado de incidencia de la acción sobre el factor, en el ámbito específico en que actúa.

Extensión (EX): representa el área de influencia del impacto en relación con el entorno.

Momento (MO): alude al tiempo que transcurre entre la aparición de la acción y el comienzo del efecto sobre el factor del medio considerado.
Persistencia (PE): se refiere al tiempo de permanencia del efecto desde su aparición.

Reversibilidad (RV): es la posibilidad de reconstruir el factor afectado por medios naturales.

Recuperabilidad (MC): se refiere a la posibilidad de reconstruir el factor afectado con intervención humana.

Sinergia (SI): contempla el reforzamiento de dos o más efectos simples, que pueden generar efectos sucesivos o que 
tienen relación, acentuando las consecuencias del impacto analizado.

Acumulación (AC): se refiere al aumento del efecto cuando persiste la causa.

Efecto (EF): se interpreta como la forma de manifestación del impacto sobre un factor como consecuencia de una acción, es decir, expresa la relación causa-efecto. Periodicidad (PR): se refiere a la regularidad de manifestación del efecto.

Los valores reunidos varían en un rango de 40 y 75 , y se clasifican en las siguientes categorías (Tabla 6):

Tabla 6. Categorías de importancia de impacto

\begin{tabular}{|c|c|}
\hline Categorías & $\begin{array}{c}\text { Valor de } \\
\text { importancia }\end{array}$ \\
\hline Impacto critico & $1>75$ \\
\hline Impacto severo & $50>1<75$ \\
\hline Impacto moderado & $25>1<50$ \\
\hline Impacto irrelevante & $1<25$ \\
\hline
\end{tabular}

Fuente: Conesa (1997)

Por último se construye la matriz resultante de doble entrada, en cuyas columnas se incorporan las acciones impactantes y en las filas los factores medioambientales susceptibles de recibir impactos. Una vez aplicada la fórmula, se obtiene el grado de transformación y el grado de impacto de cada factor, donde se pueden establecer las categorías de Importancia de Impacto (Tabla 7).

\section{Diagnóstico}

Se reconoce que las acciones más impactantes responden a los usos del suelo, los cuales alteran en mayor o menor medida el conjunto de factores ambientales restantes: degradación de tierras y calidad del paisaje.

La presión ejercida por la agricultura y la búsqueda de máxima rentabilidad en menores plazos, impulsó una actividad agropecuaria de alto impacto en los suelos no aptos para una producción agropecuaria intensiva. El avance sobre la vegetación originaria (biodiversidad) y el posterior manejo de la producción agrícola-ganadera, son la causa de la pérdida de estructura y de la disminución de fertilidad de los perfiles edáficos de la región.

Otra acción crítica es la desertificación, pues, si bien evidencia un extenso periodo de sequía (2005-2009), posiblemente por efecto natural, las acciones incontroladas en los usos del suelo han agravado la erosión eólica e impactado notablemente sobre este factor.

En cuanto a las presiones demográficas y expansión de centros urbanos, el crecimiento poblacional que experimentan las localidades del Valle Bonaerense del Río Colorado en las tres últimas décadas, indica la expansión de la principal trama productiva de la región. Se manifiesta en el proceso migratorio que se concentra en las principales localidades (Hilario Ascasubi, Villalonga y Pedro Luro), asociado con inmigrantes de origen 
Tabla 7. Matriz de importancia de impacto

\begin{tabular}{|c|c|c|c|c|c|c|c|c|c|c|c|}
\hline \multirow{3}{*}{\multicolumn{2}{|c|}{ FACTORES DEL MEDIO }} & \multicolumn{10}{|c|}{$\begin{array}{l}\text { ACCIONES VINCULADAS CON } \\
\text { LA ACTIVIDAD PRODUCTIVA }\end{array}$} \\
\hline & & \multirow{3}{*}{$\begin{array}{l}\text { UIP } \\
30\end{array}$} & \multicolumn{3}{|c|}{ Usos del suelo } & \multirow{3}{*}{ 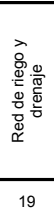 } & \multirow{3}{*}{ 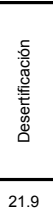 } & \multirow{3}{*}{ 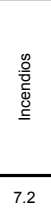 } & \multirow{3}{*}{ 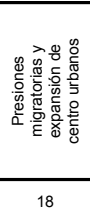 } & \multirow{3}{*}{ 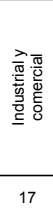 } & \multirow{3}{*}{ 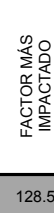 } \\
\hline & & & 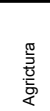 & 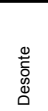 & 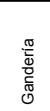 & & & & & & \\
\hline Suelos & Degradación & & 15 & 15.9 & 14.5 & & & & & & \\
\hline Agua & Calidad del agua de riego & 15 & 9 & - & - & 9.6 & 7.5 & - & - & - & 26.1 \\
\hline \multirow{5}{*}{$\begin{array}{l}\text { Condiciones } \\
\text { climáticas }\end{array}$} & Vientos & \multirow{5}{*}{25} & 18 & 18.5 & - & - & 18.5 & 8.7 & - & - & 63.7 \\
\hline & Precipitaciones & & 16 & & - & - & 19 & 9.2 & 16 & - & 60.2 \\
\hline & Temperatura & & - & - & - & - & - & 8.2 & - & - & 8.2 \\
\hline & Heladas & & 14.7 & - & - & - & - & - & - & - & 14.7 \\
\hline & Sequías & & 18 & - & 11 & - & 19 & 14.7 & - & - & 62.7 \\
\hline \multicolumn{2}{|c|}{ Biodiversidad $^{1}$} & 5 & 3.8 & 1.8 & 1.55 & - & 3.2 & 2.4 & 3.4 & - & 16.1 \\
\hline \multicolumn{2}{|l|}{ Paisaje } & 25 & 19 & 18.5 & 7.75 & 19 & 19 & 9 & 10.75 & 11.5 & 114.7 \\
\hline \multicolumn{2}{|c|}{ GRADO DE TRANSFORMACIÓN } & 100 & 113.5 & 54.7 & 34.3 & 47.6 & 108.1 & 59.4 & 32.155 & 28.5 & \\
\hline
\end{tabular}

1. Monte nativo

2. Galpones de empaque

Fuente: Elaboración propia basada en Conesa (1997).

boliviano que operan como mano de obra temporaria o golondrina durante el período de cosecha.

En lo referente al factor ambiental, se reconocen las transformaciones del paisaje natural a favor de la generación de neoecosistemas. Estas acciones muestran un estado perturbador hacia el ecosistema nativo en forma sostenible, desde hace más de un siglo. De allí que todos los componentes visibles del paisaje de la región se hayan modificado, pero, aún más, los procesos ecológicos que responden a interacciones no perceptibles y que encierren la clave de la estructura $\mathrm{y}$ apariencia del mismo.

Con respecto a la red de riego y el drenaje, el agua de riego representa tenores salinos superiores a los valores permitidos, el balance salino expone un valor positivo con un importante lavado de sales de los suelos.

En lo relativo a los incendios en el Valle Bonaerense del Río Colorado, hay que anotar que se deben a dos causas fundamentales: las naturales, a partir del desarrollo de tormentas eléctricas, y, por otro lado, a la acción del hombre causante de la mayoría de los focos ígneos. Las áreas de cultivos intensivos localizadas en el noreste y en la franja central del valle bonaerense del río Colorado, son las que presentan menor riesgo. Estos son sectores menos propensos a sufrir incendios, situación que se asocia con la mayor humedad ambiental propia de zonas con riego artificial. 
Junto al incremento de las actividades industriales y comerciales, está la producción de desechos. En especial basurales incontrolados y residuos peligrosos expuestos al aire libre en bordes de acequias y canales que por lixiviación llegan a las aguas subterráneas generando contaminación de los mismos.

En cuanto a los factores ambientales, el comportamiento de las variables se manifiesta especialmente en la incidencia de fuertes vientos y ausencia de precipitaciones, que han generado condiciones de sequía, afectando directamente los suelos y la actividad agrícola-ganadera.

Con respecto a las precipitaciones, si se compara el período 2000-2009, tanto en el partido de Patagones como en Villarino se aprecia una disminución de la precipitación en relación con el promedio histórico de la región. Durante el periodo comprendido entre 1980 y 1990, el incremento de las precipitaciones sobre la media histórica generó un corrimiento de las isohietas hacia el suroeste. Este pulso húmedo generó gran expectativa propiciando la intensificación agrícola en los productores y causando mayor presión sobre el suelo y la vegetación nativa. Esta fue una oportunidad para incrementar el número de ganado, que causó sobrepastoreo y alta demanda de forraje.

La tasa de pérdida de biodiversidad está asociada al desmonte de grandes extensiones de tierra con fines agrícolas. La modificación y pérdida de hábitats junto a la incorporación de especies exóticas, alteran el ecosistema frágil de estos ambientes áridos y semiáridos, lo que, unido a la temperatura, constituyen los factores impactados de menor valor.

En cuanto a la ganadería bajo el manejo de pastoreo, en épocas favorables de humedad, provocó un reemplazo de la vegetación natural por las pasturas y mayor presión de carga animal a lo largo del tiempo. Este sobrepastoreo fue un factor agravante en la erosión del suelo que se manifiesta en la actualidad bajo un período de crisis hídrica.

\section{Conclusiones}

La oferta natural del agua, sumada a oportunidades económicas del mercado, ha propiciado la intensificación agrícola en el Valle Bonaerense del río Colorado. En gran medida, esta coyuntura alentó la incorporación de tierras marginales al sistema productivo, que en su mayoría no contempló las prácticas agrícolas conservacionistas. Esto trajo aparejado el deterioro de los suelos vinculado al incremento de la salinización, aumento de la erosión y la progresiva disminución de la calidad de las tierras agropecuarias existentes.

La búsqueda de nuevas áreas redujo la superficie de hábitats naturales con la consecuente pérdida de biodiversidad. Estos cambios sustituyeron los ecosistemas nativos a favor de la degradación de suelos con baja respuesta a la recuperación. 
El manejo sostenible de los recursos naturales implica el conocimiento de información básica previa. Esta línea de investigación se enmarca dentro del estudio de impacto ambiental (EIA), como un proceso de análisis encaminado a identificar, predecir, interpretar, valorar, prevenir y comunicar el efecto de una acción determinada sobre el medio ambiente. La aplicación de la matriz de importancia de impacto, puede proveer una base inicial en la configuración de estudios más amplios que contemplen la gestión de estos recursos.
Por último vale advertir que las políticas ambientales deben acompañar a las políticas económicas, cuando estas tengan como finalidad lograr que el desarrollo sea sostenible y duradero. De este modo, el papel del gobierno es primordial en cuanto a orientar las políticas y la aplicación de la legislación vigente en el cuidado del ambiente. A su vez, cabe la capacitación de los actores sociales responsables de llevar a cabo transformaciones productivas que contemplen la racionalidad ambiental. 


\section{Literatura citada}

Capelli de Steffens, A. et al. (1994). La transición climática del sudoeste bonaerense. Serie: Monografías. Sigeo. Sección de Investigaciones del Departamento de Geografía. Bahía Blanca, Argentina: Universidad Nacional del Sur.

Conesa, V. (1997). Guía metodológica para la evaluación del impacto ambiental. Madrid: Mundi-Prensa.

Coscia, S. (1988). Impacto ambiental en el Valle Inferior del Río Colorado. Informe de tesis de Licenciatura en Geografía. Departamento de Geografía. Universidad Nacional del Sur, Bahía Blanca, Argentina.

Dumrauf, M. (2008). Evolución de la frontera agrícola en los Partidos de Villarino y Patagones, período 1975-2005 y su implicancia en el deterioro ambiental. Informe de tesis de Licenciatura en Geografía. Departamento de Geografía y Turismo. Universidad Nacional del Sur. Bahía Blanca, Argentina.

Estevan, M. (1989). Evaluación de impacto ambiental. Madrid: MAPFRE.

Pezzola, A. \& Winschel, C. (2002). Evaluación de la superficie afectada por incendios en el Partido de Villarino y Patagones, Provincia de Buenos Aires, 2001/2002. Laboratorio de Teledetección y SIG. Instituto Nacional de Tecnología Agropecuaria.

Pezzola, A. \& Winschel, C. (2004). Estudio espacio temporal de incendios rurales, utilizando percepción remota y SIG. Boletín Técnico, (20). Instituto Nacional de Tecnología Agropecuaria.

Pezzola, A. \& Agamennonni, R. (2009). Estimación expeditiva de suelos erosionados en el partido de Patagones- Provincia de Buenos Aires. Instituto Nacional de Tecnología Agropecuaria.

Pezzola, A. \& Ramos, B. (2005). Organización espacial del partido de Villarino. Provincia de Buenos Aires. Boletín Técnico, (21). Instituto Nacional de Tecnología Agropecuaria.

Sánchez, R. \& Pezzola, A. (1998). Caracterización edafoclimática del área de influencia del INTA. EEA. H. Ascasubi. Partidos de Villarino y Patagones. Provincia de Buenos Aires. Boletín de divulgación, (18).Instituto Nacional de Tecnología Agropecuaria.

Soler, M. (1997). Manual de gestión del medio ambiente. Barcelona: Ariel. 